\title{
Alprazolam Dependence - The Sting in the Tail: A Case Report
}

\author{
JS Martin, WD Abel, CA Sewell
}

\begin{abstract}
This is a case report of a 38-year old female seen in the emergency department after ingesting a total of 99 alprazolam tablets amounting to approximately $50 \mathrm{mg}$ of alprazolam over the previous two days. The patient admitted to taking between $7.5 \mathrm{mg}$ and $15 \mathrm{mg}$ of alprazolam on a daily basis over the last three months. Though formally diagnosed with bipolar I disorder in her early twenties, she had never consistently taken the mood stabilizers prescribed for her condition as the associated weight gain made her feel fat and unattractive. Alprazolam in increasing doses was needed by the patient to quieten her symptoms, and in an effort to accumulate large numbers of same, she obtained the drug by visiting numerous physicians or by purchasing it illegally. The patient has a strong family history of both suicide and bipolar I disorder. The patient admitted to three suicide attempts, two of which involved the drug alprazolam.
\end{abstract}

Keywords: Alprazolam dependence, bipolar disorder, suicide

WIMJ Open 2015; 2 (1): 52

\section{INTRODUCTION}

Since their introduction in the 1950s, benzodiazepines continue to be a popular item on most prescriptions. In the 1970s, benzodiazepines, as a class, became the most commonly prescribed of all drugs in the world (1). This was not surprising as the benzodiazepines were marketed and Food and Drug Administration (FDA) approved as possessing a wide range of actions spanning sedative/hypnotic, anxiolytic, anti-convulsant and muscle relaxant abilities with a low potential for toxicity and dependence. As such, these "harmless" drugs were prescribed by both psychiatrists and non-psychiatrists alike for complaints such as anxiety, depression, insomnia and even ordinary life stressors $(2,3)$. Unfortunately, to quote Professor of Psychiatry, Dr Heather Ashton, like the proverbial scorpion, these drugs would soon reveal the sting in their tail $(1,2)$. By the 1980s, long-term benzodiazepine users reported that the drugs became less efficacious over time, requiring larger doses to attain the same effect. In addition, these same users also bemoaned the fact that they were unable to decrease or cease use of these drugs without experiencing adverse withdrawal effects. Dependence, the sting in the tail of the popular benzodiazepines, had become apparent.

To date alprazolam is the most prescribed and misused benzodiazepine on the retail market in the United States of America (USA), surpassing even the ever popular antidepressant medications $(2,4)$. Of the 250 million prescriptions written for psychiatric drugs in the USA in 2009 , nearly

From: Department of Community Health and Psychiatry, The University of the West Indies, Kingston 7, Jamaica, West Indies.

Correspondence: Professor WD Abel, Department of Community Health and Psychiatry, The University of the West Indies, Kingston 7, Jamaica, West Indies. E-mail: drwendelabel@gmail.com
50 million prescriptions, that is, more than one per second, were written for alprazolam. In fact, it is estimated that $11 \%-15 \%$ of all adult Americans have a bottle of alprazolam in their medicine cabinet $(2,4-6)$. The popularity of Xanax, one of the trade names of alprazolam, has been underscored by the fact that it is ranked number nine on the list of the topearning drugs in the USA (6). This statistic is even more remarkable given the fact that, in addition to Xanax, there are many generic forms which, if taken into account, could escalate the ranking even higher (4).

Alprazolam, trade name Xanax, the drug at the centre of this case report, is a short-acting anxiolytic of the benzodiazepine class. It was first released in 1981 (7). Alprazolam, like other benzodiazepines, binds to specific sites on the $\mathrm{GABA}_{\mathrm{A}}$ gamma-amino-butyric acid receptor. Alprazolam is commonly used and FDA approved for the medical treatment of panic disorder, and anxiety disorders, such as generalized anxiety disorder or social anxiety disorder. However, given its sedative/hypnotic properties, it is also used as a treatment for both chronic and acute insomnia. It has a fast onset of action and symptomatic relief. Ninety per cent of peak effects are achieved within the first hour (although onset may begin at 8-25 minutes of ingestion).

While it is controversial as to whether tolerance will develop to the anxiolytic/anti-panic effects of alprazolam (8), it is reported that tolerance will, however, develop to the sedative-hypnotic effects within a couple of days (9). Withdrawal symptoms or rebound symptoms may occur after ceasing treatment abruptly following a few weeks or longer of steady dosing, and may necessitate a gradual dose reduction $(10,11)$. 


\section{CASE REPORT}

A 38-year old female was sent to the emergency department of a private hospital in Kingston, Jamaica, by her private psychiatrist after she admitted to ingesting thirty-one $0.5 \mathrm{mg}$ alprazolam tablets on the day of her visit, as well as a total of sixty-eight $0.5 \mathrm{mg}$ alprazolam tablets on the previous day. She was transferred to the psychiatric unit of the University Hospital of the West Indies for further investigations and definitive management. On admission, the patient reported that she had suffered from mood swings, which she described as feelings of sadness and tearfulness alternating with feelings of anger, irritability or unwarranted elation since she was a teenager, although she was not formally diagnosed with bipolar I disorder until her early twenties.

Since the time of her initial diagnosis, the patient stated that she had been prescribed quetiapine, alprazolam and sodium valproate in varying dosages by numerous psychiatrists and general practitioners. However, she admitted that since diagnosis, she has never taken the prescribed quetiapine or the sodium valproate consistently or at the prescribed doses as, in addition to other symptoms, the associated weight gain made her feel fat and unattractive. She reported that she has instead used alprazolam in increasing dosages to ameliorate her symptoms. The patient reported that of late, the alprazolam seemed to be less effective than in the past as she now "feels nothing" even with extremely high doses.

The patient informed the clinician that she had been taking between 15 and 30 alprazolam tablets $(0.5 \mathrm{mg})$ on any given day over the last three months and that, given her need for increased doses of alprazolam, she had resorted to sourcing the alprazolam by attending the offices of various general practitioners and psychiatrists whenever the amount prescribed by her personal psychiatrist was exhausted. The patient also reported that she supplemented her alprazolam stock by purchasing alprazolam illegally on the "streets" at exorbitant prices.

Though the patient admitted to three occasions of attempted suicide - the last occasion being three months ago - at the time of admission, she denied that this present occasion of alprazolam overdose was a suicide attempt. She reported that since her last suicide attempt, she had vowed not to leave her four children motherless. The patient insisted that she took the tablets to get some sleep and "peace of mind". She further revealed that she had a non-therapeutic abortion one year previously and that since then she has been feeling either angry, irritable and sad or elated with consistently high energy levels, necessitating increasing doses of alprazolam to assuage her feelings of guilt.

Additionally, the patient admitted to uncharacteristically "channelling a lot of unkind things" in her head about her husband which has resulted in a breakdown of their relationship. The patient is a teacher at a high school and is currently pursuing a Master's degree. The patient denies use of alcohol or any illicit substance of abuse. Significantly, the patient's mother was diagnosed with bipolar I disorder and her paternal aunt died by suicide.

All blood and radiological and ultrasound investigations were returned as normal. In particular, no renal or hepatic abnormalities were noted. Sodium valproate and quetiapine were the mood stabilizers prescribed for the patient and the alprazolam was replaced with diazepam in an effort to treat her alprazolam dependence and avoid withdrawal side effects.

After a thorough psychological evaluation, daily psychotherapy was commenced. Unfortunately, after one week on the ward, and in spite of a detailed family meeting with the patient's husband at which time the implications of his wife's alprazolam dependent state was outlined to him, the patient's husband discharged her against medical advice stating that the patient needed to return home to attend to their children and to resume her studies. The patient did not attend the scheduled ward reviews and when contacted stated that she had chosen to continue her care elsewhere.

\section{DISCUSSION}

Prevention of alprazolam dependence can be minimized by rigid adherence to official usage guidelines for the drug which state that prescriptions should be restricted to shortterm use. Alprazolam should only be prescribed for intermittent or occasional use, which may be extended to a twoto four-week duration, if needs be (12). Benzodiazepines are not indicated for the long- term treatment of any medical or psychiatric condition. Special precaution should be taken when prescribing for chronic psychiatric conditions such as bipolar disorder, schizophrenia, anxiety disorders and chronic insomnia. Cases like the one outlined mandate that all medical practitioners including psychiatrists examine the risk-benefit ratio of all patients to whom they wish to prescribe alprazolam. This analysis should be undertaken at the initial consultation in an effort to ascertain and weigh the risk of subsequent dependence against the therapeutic value of the drug (13).

With respect to this patient, it is clear that not enough attention was paid to her strong family history of bipolar disorder and suicide by either the general practitioners or psychiatrists with whom she came into contact. Finally, the overprescribing of alprazolam, and other benzodiazepines in general, has resulted in a large, unwarranted population of long-term alprazolam users. The result of which is drug dependence and leakage of alprazolam and other benzodiazepines into the illicit drug market.

\section{REFERENCES}

1. Ashton H. Benzodiadepine dependence. In Haddad P, Dursun S, Deakin B, eds. Adverse symptom and psychiatric drugs. Oxford: Oxford University Press; 2004: 239-60.

2. Petursson H, Lader MH. Benzodiazepine dependence. Br J Addict 1984: 76: 133-46.

3. Mandrioli R, Mercolini L, Raggi MA. Benzodiazepine metabolism: an analytical perspective. Curr Drug Metab 2008; 9: 827-44. doi: $10.2174 / 138920008786049258$. 
4. Langreth R, Herper M. In Pictures: the most popular prescription drugs. Forbes; 2010.

5. US Department of Health and Human Services. Drug Abuse Warning Network, 2006: national estimates of drug-related emergency department visits. Rockville, MD: Substance Abuse and Mental Health Services Administration; 2008 [cited 2012 Feb 13]. Available from: http://archive.samhsa.gov/data/dawn/files/ED2006/DAWN2k6ED.pdf

6. Armstrong W. The truth about Whitney Houston and Xanax. Huffington Post; 2012. Available from: http://www.huffingtonpost. com/2012/02/15/whitney-houston-xanax_n_1279947.html

7. Walker S. A dose of sanity: mind, medicine, and misdiagnosis. New York: John Wiley and Sons; 1996: 64-65.

8. Moylan S, Giorlando F, Nordfjærn T, Berk M. The role of alprazolam for the treatment of panic disorder in Australia. Aust $\mathrm{N} \mathrm{Z} \mathrm{J}$ Psychiatry 2012; 46: 212-24. doi: 10.1177/0004867411432074.

9. Pavuluri MN, Janicak PG, Marder SR. Principles and practice of psychopharmacotherapy. $5^{\text {th }}$ ed. Philadelphia, PA: Wolters Kluwer Health/Lippincott Williams and Wilkins; 2010: 535.

10. Vester JC, Volkerts ER. Clinical pharmacology, clinical efficacy, and behavioral toxicity of alprazolam: a review of the literature. CNS Drug Reviews 2004; 10: 45-76. doi: 10.1111/j.1527-3458.2004.tb00003.x.P

11. Galanter M. The American Psychiatric Publishing Textbook of Substance Abuse Treatment. $4^{\text {th }}$ ed. Washington, DC: American Psychiatric Publishing; 2008: 222.
12. Committee on Safety of Medicines. Benzodiazepines, dependence and withdrawal symptoms. Current Problems 1988; (21): 1-2.

13. Royal College of Psychiatrists. Benzodiazepines: risk, benefits and dependence: a re-evaluation. Council Report CR 59. London: Royal College of Psychiatrists; 2002.

Submitted 13 Oct 2014

Accepted 27 Oct 2014

Published 16 Mar 2015

Online: http://www.mona.uwi.edu/wimjopen/article/1620

(C) Martin et al 2015

This is an open access article made freely available under Creative Commons Attribution 4.0 International (CC BY 4.0). Users are free to share, copy and adapt this work as long as the copyright holder (author) is appropriately and correctly credited. See http:// creativecommons.org/licences/by/4.0/deed.en_us for more information. 